\title{
Pentoxifylline as Adjuvant Therapy to Etanercept in Patients with Moderately to Highly Active Rheumatoid Arthritis
}

\author{
Samer I. Mohammed ${ }^{1, *}$, Faiq I. Gorial ${ }^{2}$, Ibrahim A. Majeed $^{1}$ \\ ${ }^{1}$ Department of Clinical Pharmacy, College of Pharmacy, University of Baghdad, Baghdad, Iraq \\ ${ }^{2}$ Department of Medicine, College of Pharmacy, University of Baghdad, Baghdad, Iraq \\ *Corresponding author: samerpharma70@gmail.com
}

Received June 26, 2013; Revised August 16, 2013; Accepted August 19, 2013

\begin{abstract}
Objective: Rheumatoid arthritis (RA) is a common inflammatory disease associated with many extraarticular features. The aim of the study was to evaluate the effects of pentoxifylline (PTX) as adjuvant therapy to etanercept in moderately to highly active RA. Methods: A single center randomized double-blind placebocontrolled trial of 8 weeks duration was performed. Disease activity was measured via calculating the disease activity score in 28 joints using erythrocyte sedimentation rate (DAS28-ESR) and by simplified disease activity score in 28 joints using hsCRP (SDAI-CRP). 40 Patients who were using etanercept (ETN) were randomly allocated to receive each day either pentoxifylline $400 \mathrm{mg}$ tablet twice daily or capsules prefilled with glucose as placebo also twice daily and were evaluated at baseline and at week 8 for clinical and hematological parameters. Results: Tumor necrosis factor (TNF), high sensitive C-reactive protein (hsCRP), duration of morning stiffness, and cardiovascular risk were significantly more reduced in pentoxifylline group than placebo group after 8 weeks. Non significant changes were observed in clinical parameter like swelling joints counts (SJC),tender joints counts(TJC),visual analogue scale(VAS),evaluator global assessment (EGA),DAS28-ESR, SDAI-CRP and hematological parameter like hemoglobin $(\mathrm{Hb})$ amount, erythrocyte sedimentation rate (ESR) and white blood cells (WBC) count between groups. Conclusion: PTX significantly decreased pro-inflammatory markers (TNF, hsCRP), duration of morning stiffness and cardiovascular risk. This suggests that pentoxifylline may be a promising and useful strategy to reduce the systemic inflammation and cardiovascular morbidity and mortality observed in RA patients.
\end{abstract}

Keywords: rheumatoid arthritis, pentoxifylline, disease activity

Cite This Article: Samer I. Mohammed, Faiq I. Gorial, and Ibrahim A. Majeed, "Pentoxifylline as Adjuvant Therapy to Etanercept in Patients with Moderately to Highly Active Rheumatoid Arthritis." American Journal of Pharmacological Sciences 1, no. 4 (2013): 61-66. doi: 10.12691/ajps-1-4-4.

\section{Introduction}

Rheumatoid arthritis (RA) is a chronic systemic autoimmune inflammatory disease that affects all ethnic groups throughout the world [1] with increased morbidity and mortality from premature cardiovascular disease (CVD). Up to $50 \%$ of this excess mortality is secondary to ischemic heart disease (IHD) closely followed by cerebrovascular disease [2], with a 1.5-fold increase in the standardized mortality ratio due to $\mathrm{CV}$ events compared with the general population [3].

The principle inflammatory cytokines that believed to have a key position in the pathogenesis of RA include TNF- $\alpha$ and IL-6, both of which are targets for therapy [4].

Pentoxifylline is a tri-substituted xanthine derivative designated chemically as 3, 7-Dihydro-3, 7-dimethyl-1-(5oxohexyl)-1H-purine-2, 6-dione that, unlike theophylline, is a hemorheologic agent, i.e., an agent that affects blood viscosity [5]. Pentoxifylline is frequently prescribed for patients with peripheral arterial disease, mainly because of its capacity to deform hemocytes and to its vasodilatation effect [6]. This drug can also decrease levels of tumoral necrosis factor, an important inflammatory cytokine responsible for the increased endothelial expression of adhesion molecules and for IL-6 production [7]. The precise mode of action of pentoxifylline and the sequence of events leading to clinical improvement are still to be defined [5].

Many studies have demonstrated the possible antiinflammatory effect of pentoxifylline (PTX). A prospective 3-month open evaluation of PTX in a group of adult patients with RA refractory to conventional disease curative therapies in 1995 where 19 patients with RA assessed clinically according to the World Health Organization/International League of Associations for Rheumatology (WHO/ILAR) criteria at baseline, and 3 months after the initiation of therapy. They evaluated a complete blood count, erythrocyte sedimentation rate (ESR), and whole blood assays of TNF- $\alpha$ production. After 3 months they found a significant diminution in number of tender and swollen joints as well as the ESR (p $<0.05$ ) although no consistent effects on TNF- $\alpha$ 
production were observed. Furthermore, whole blood assays of TNF-alpha production shortly after initiation of pentoxifylline therapy were not predictive of the clinical response to this agent and they concluded that although pentoxifylline may possess therapeutic properties in RA, any beneficial effects appear to be unrelated to changes in TNF- $\alpha$ generation in whole blood assays [8].

Also Dysregulation of TNF- $\alpha$ production which thought to be important in rheumatoid arthritis was tested in 1995 by using combination of PTX and thalidomide in an open study of rheumatoid arthritis patients to assess the effect on TNF production, the antiarthritic effects and toxicity of this combination, in which 12 patients with active rheumatoid arthritis were treated with 1200 mg pentoxifylline and $100 \mathrm{mg}$ thalidomide daily for 12 weeks. The results showed a lowering in the TNF production capacity during treatment $(\mathrm{P}<0.03)$ whereas production capacity of IL- 6, IL-10, and IL-12 was not affected. They concluded that although pentoxifylline/thalidomide reduced the production capacity of TNF, the benefit/side effects ratio was poor due to multiple adverse effects, while clinical observation suggests limited efficacy [9].

Another study on a 64 year-old man with seronegative RA who had 23 swollen joints, 32 painful joints, and ESR $135 \mathrm{~mm} / \mathrm{h}$. All these parameters were dramatically improved 3 weeks after administration of PTX $300 \mathrm{mg} / \mathrm{d}$ and prednisolone $5 \mathrm{mg} / \mathrm{d}$. Discontinuation of PTX in this patient resulted in rapid exacerbation of RA and when PTX was restarted the patient showed complete recovery from arthritis with normalization of ESR within 3 months and was maintained a complete remission for another 1 year. This case further supports a potential antirheumatic effect of PTX on some patients with RA [10].

Also, in an animal model, Al-Saad et al evaluated the dose-response relationship of the anti-inflammatory activity of PTX in experimental animal models of chronic inflammation in 2012 and found that PTX in a dose dependent pattern attenuates formaldehyde-induced chronic inflammation and cotton-pellet induced granuloma in rats and potentiates the anti-inflammatory activity of dexamethasone and methotrexate [11]. The aim of this study was to evaluate the effects of PTX as adjuvant therapy to etanercept in moderately to highly active RA.

\section{Subjects and Methods}

\subsection{Study Design}

This was an 8-week randomized double blind placebo controlled single center trial conducted at Rheumatology Unit, Baghdad Teaching Hospital, Baghdad, Iraq carried out over 5 months from September 2012 till March 2012 at Rheumatology Unit, Baghdad Teaching Hospital.

Patients were randomly allocated to receive each day either pentoxifylline400 mg tablet twice daily or capsules prefilled with glucose as placebo (PBO) orally also twice daily in addition to their weekly subcutaneous etanercept dose. Pentoxifylline was bought from Sanofi-AventisEgypt Company, Egypt. Whereas glucose was bought from SDI, Samarra, Iraq. Patients were evaluated at baseline and at week 8 .

Informed consent was obtained from all participants and this study was approved by the ethical committee of
Baghdad University, College of Medicine - Medical Department.

\subsection{Sample Selection}

Eligible patients had confirmed RA according to the 1987 American College of Rheumatology (ACR) criteria with moderate to highly active disease defined as disease activity score based on 28 joints and ESR (DAS28-ESR) greater than 3.2 at baseline. For inclusion, patients also were required to have taken etanercept subcutaneously and regularly for at least 4 previous consecutive months without any clinical response. The exclusion criteria included presence of contraindication to PTX (Cerebral hemorrhage, extensive retinal hemorrhage, acute myocardial infaction, and sever cardiac arrhythmia), renalimpairment, hepatic impairment, pregnancy, breast feeding, patients already onPTX, patients with mild or inactive RA,using DMARDs other than etanercept , with high dose of NSAIDS, and patients with co-existent other connective tissue disease. Additionally, 20 healthy age and sex matched individuals were considered as a control group.

\subsection{Clinical and Laboratory Evaluation}

Clinical evaluation of patients for tender and swelling joints was done by specialized rheumatologist who was blinded to treatment at zero time (baseline) and after 8 weeks. The RA disease activity was measured using DAS28-ESR) [12] and Simplified Disease Activity Index (SDAI) [13]. DAS28 is calculated from the number of tender and swollen joint counts (TJC and SJC; 28-joint count), patient self-assessment of disease activity (visual analog scale, VAS), and erythrocyte sedimentation rate (ESR.]. SDAI is the numerical sum of five outcome parameters: TJC and SJC (based on a 28-joint assessment), patient and physician global assessment of disease activity (VAS $0-10 \mathrm{~cm}$ ) and level of $\mathrm{C}$ reactive protein (CRP).DAS28 and SDAI can be calculated according to the following formula:

DAS28 $=0.56($ TJC) $0.5+0.28(\mathrm{SJC}) 0.5+0.70 \ln (\mathrm{ESR})$ +0.014 (VAS)

$\mathrm{SDAI}=\mathrm{TJC}+\mathrm{SJC}+\mathrm{EGA}+\mathrm{VAS}+\mathrm{CRP}$

Blood specimen collection and laboratory analysis (at baseline and after 8 weeks) of WBCs count, ESR and Hb, high sensitive CRP (hsCRP),TNF- $\alpha$, was done by specialized laboratory researchers who did not participate in this study. WBCs count and Hb were measured by a hematology auto-analyzer (Ruby-CELL-DYN 08H56-02, Abbott Company, Abbott Park, IL, USA). ESR was measured by Westergren method [10eh].hsCRP [14] and TNF- $\alpha$ [15] by using ELIZA technique.

\subsection{Statistical Analysis}

Statistical software (SPSS version 16, Chicago, IL, USA) was used for data input and analysis. Continuous variables were presented as mean \pm standard deviation (SD) and discrete variables were presented as numbers and frequencies. Chi square test for independence was used to test the significance of association between discrete variables. Continuous variables were tested by the Shapiro Wilk test to determine if they were normally or abnormally distributed. ANOVA test was used to test the 
significance of difference in the mean of 3 independent samples in normally distributed continuous variables. Unpaired t-test was used to test the significance of difference in the mean of two independent samples in normally distributed continuous variables and Mann Whitney test for abnormally distributed data. Findings with $\mathrm{P}$ value less than 0.05 were considered significant whereas $\mathrm{P}$ values less than 0.01 considered highly significant.

\section{Results}

Of a total of 49 patients who were randomized in this double-blind study, 40 completed the 8 weeks of treatment (20 from the pentoxifylline (PTX) group and 20 from the placebo) (Figure 1).

The mean age of PTX group did not differ significantly from placebo and controls(50.25 \pm 9.91 years vs 49.8 \pm 9.84 years vs $42.05 \pm 9.88$ years, $\mathrm{P}>0.05$ ) respectively. Also there was no statistical significant difference in the female to male ratio among PTX group, placebo group, and controls (15:5 (75\%)vs12:8 (60\%) vs10:10(50\%), $\mathrm{P}>0.05$ ) respectively (Table 1 ).

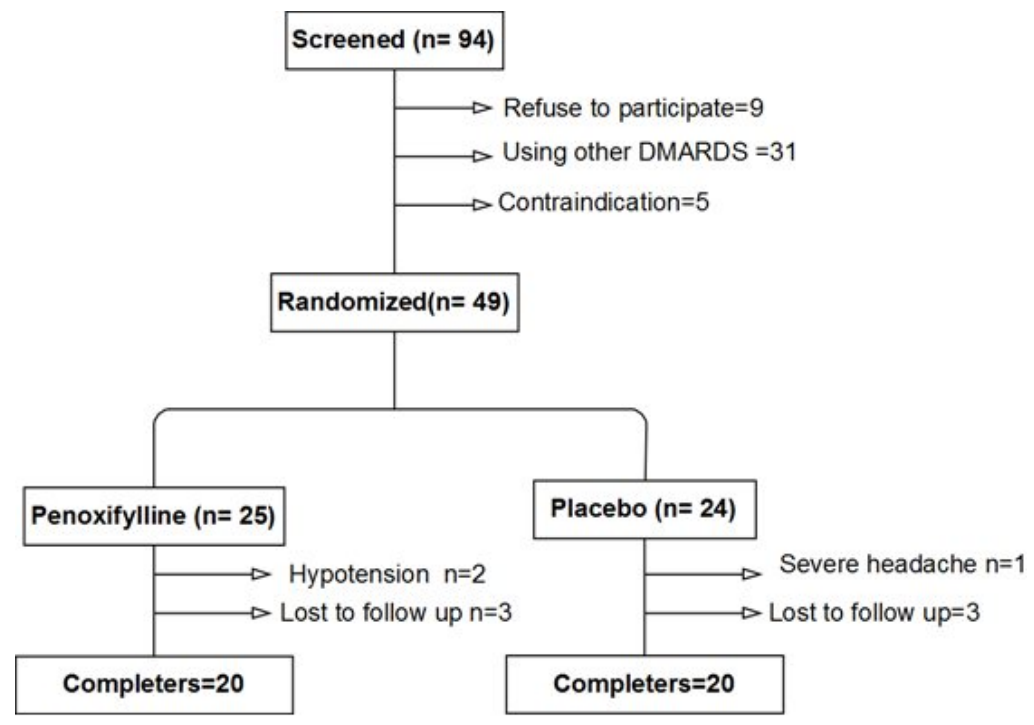

Figure 1. Total number of patients participated in the current study

Table 1. Baseline characteristics of patients and controls

\begin{tabular}{|c|c|c|c|c|}
\hline Parameter & $\begin{array}{c}\text { Pentoxifylline } \\
(\mathrm{n}=20)\end{array}$ & $\begin{array}{l}\text { Placebo } \\
(n=20)\end{array}$ & $\begin{array}{l}\text { Control } \\
(\mathrm{n}=20)\end{array}$ & P-value \\
\hline Age inyears(mean \pm SD) & $50.25 \pm 9.91$ & $49.8 \pm 9.84$ & $42.05 \pm 9.88$ & 0.07 \\
\hline Female:Male [n(\%)] & $15: 5(75 \%)$ & $12: 8(60 \%)$ & $10: 10(50 \%)$ & 0.66 \\
\hline Smoking[n(\%)] & $2(10 \%)$ & $0(0 \%)$ & $5(25 \%)$ & 0.189 \\
\hline Duration ofdisease inyears (mean \pm SD) & $11.95 \pm 8.82$ & $7.5 \pm 5.50$ & & 0.068 \\
\hline Familyhistoryof rheumatoidarthritis[n](\%)] & $3(15 \%)$ & $3(15 \%)$ & & 0.87 \\
\hline Diseaseactivityscore of 28joints (mean \pm SD) SD) & $5.73 \pm 1.27$ & $5.59 \pm 0.94$ & & 0.971 \\
\hline Subcutaneousnodules & $3(15 \%)$ & $4(20 \%)$ & & 0.243 \\
\hline Positive Rheumatoidfactor [n(\%)] & $15(75 \%)$ & $17(85 \%)$ & & 0.342 \\
\hline
\end{tabular}

Continuous variables presented as Mean \pm Standard deviation; and discrete variables as numbers and frequencies.

Baseline hematological parameters showed that $\mathrm{Hb}$ level was significantly lower in patients with active RA than healthy control subjects $(\mathrm{P}<0.05)$. Whereas ESR and WBC count were significantly higher in patients with active RA than those in control group ( $\mathrm{P}<0.001$, Table 2 ).
Table 3 showed no significant difference in the baseline clinical parameters between PTX and placebo group patients $(\mathrm{P}>0.05)$.

After 8 weeks of starting adjuvant treatment with eitherPTX or placebo, we found that only hsCRP and TNF- $\alpha$ decreased significantly by PTX (P < 0.05, Table 4$)$.

Table 2. Baseline hematological parameters of the patients and controls

\begin{tabular}{|c|c|c|c|c|}
\hline Parameter & $\begin{array}{c}\text { Pentoxifylline } \\
(\mathrm{n}=20)\end{array}$ & $\begin{array}{c}\text { Placebo } \\
(\mathrm{n}=20)\end{array}$ & $\begin{array}{l}\text { Control } \\
(n=20) \\
\end{array}$ & P-Value \\
\hline ESR (mm/hr.) & $66.05 \pm 37.20$ & $65.45 \pm 31.48$ & $7.12 \pm 3.37$ & $<0.001$ \\
\hline Hemoglobin(g/dl) & $12.24 \pm 1.23$ & $12.29 \pm 1.34$ & $14.05 \pm 1.01$ & $<0.05$ \\
\hline WBC count (Nano Liter) & 10.182.35 & $9.92 \pm 2.44$ & $5.33 \pm 0.89$ & $<0.001$ \\
\hline hsCRP(mg/dl) & $8.37 \pm 3.60$ & $8.19 \pm 3.23$ & $4.25 \pm 3.16$ & $<0.001$ \\
\hline TNF- $\alpha(\mathrm{pg} . / \mathrm{ml})$ & $66.18 \pm 45.89$ & $42.92 \pm 37.42$ & $7.39 \pm 2.90$ & $<0.001$ \\
\hline
\end{tabular}

Continuous variables presented as Mean \pm Standard deviation 
Table 3. Baseline clinical parameters of the patients

\begin{tabular}{|c|c|c|c|}
\hline Parameter & Pentoxifylline $(n=20)$ & Placebo $(n=20)$ & P-Value \\
\hline Tender joint count (TJC) & $9.90 \pm 5.17$ & $8.75 \pm 4.09$ & 0.478 \\
\hline Swelling joint count (SJC) & $3.90 \pm 2.45$ & $3.00 \pm 2.18$ & 0.478 \\
\hline Visual analogue scale (VAS) & $55.50 \pm 16.38$ & $51.50 \pm 18.43$ & 0.929 \\
\hline Evaluator global assessment (EGA) & $5.23 \pm 1.61$ & $4.80 \pm 1.96$ & 0.499 \\
\hline RA disease activity as measured by DAS28 & $5.73 \pm 1.27$ & $5.59 \pm 0.94$ & 0.971 \\
\hline RA disease activity as measured by SDAI & $27.71 \pm 9.46$ & $25.105 \pm 7.73$ & 0.541 \\
\hline Duration of morning stiffness(min) & $17.85 \pm 16.63$ & $12.75 \pm 13.78$ & 0.180 \\
\hline
\end{tabular}

Continuous variables presented as Mean \pm Standard deviation

Table 4. Changes inhematological parameters after 8 weeks

\begin{tabular}{|c|c|c|}
\hline Parameter & Pentoxifylline (n=20) & Placebo (n= 20) \\
\hline ESR (mm/hr.) & $-16.50 \pm 14.38$ & $-18.05 \pm 18.13$ \\
\hline Hemoglobin(g/dl) & $-0.17 \pm 0.73$ & $+0.10 \pm 1.70$ \\
\hline WBC count (Nano Liter) & $-0.85 \pm 0.93$ & $-0.74 \pm 1.11$ \\
\hline hsCRP(mg/dl) & $-0.66 \pm 1.13$ & $-0.12 \pm 0.58$ \\
\hline TNF- $\alpha$ (pg./ml) & $-18.06 \pm 18.99$ & $-3.58 \pm 7.46$ \\
\hline
\end{tabular}

Continuous variables presented as Mean \pm Standard deviation.ESR= Erythrocyte sedimentation rate.

$\mathrm{WBC}=$ white blood cells. HsCRP=High sensitive $\mathrm{C}$ - reactive protein.

TNF- $\alpha=$ Tumor necrosis factor $\alpha$ * Significantly different compared to placebo $(\mathrm{p}<0.05)$

Table 5. Changes inclinical parameters after 8weeks

\begin{tabular}{|c|c|c|}
\hline Parameter & Pentoxifylline $(\mathrm{n}=20)$ & Placebo $(\mathrm{n}=20)$ \\
\hline Tender joint count (TJC) & $-2.3 \pm 1.75$ & $2.35 \pm 2.58$ \\
\hline Swelling joint count (SJC) & $-0.6 \pm 1.14$ & $-0.35 \pm 1.84$ \\
\hline Visual analogue scale (VAS) & $-10.8 \pm 9.77$ & $-5.25 \pm 10.94$ \\
\hline Evaluator global assessment (EGA) & $-0.90 \pm 1.01$ & $-0.78 \pm 1.61$ \\
\hline RA disease activity as measured by DAS28 & $-0.60 \pm 0.61$ & $-0.81 \pm 0.88$ \\
\hline RA disease activity as measured by SDAI & $-4.58 \pm 2.94$ & $-4.42 \pm 5.29$ \\
\hline Duration of morning stiffness(min) & $-7.45 \pm 9.05$ & 0.305 \\
\hline
\end{tabular}

Continuous variables presented as Mean \pm Standard deviation. * Significantly different compared to placebo $(\mathrm{p}<0.05)$.

In addition, only duration of morning stiffness decreased significantly by PTX $(\mathrm{P}<0.05)$ while other parameters showed no significant difference between the effect of PTX and placebo (P > 0.05, Table 5).

\section{Discussion}

Swelling joints are considered as the best single variable that detects response to drug therapy in RA patients [16]. The present study showed that PTX reduced swelling joints significantly while placebo did not produce any significant change after 8weeks of therapy, but overall there was non significant difference between the effect of PTX and placebo on SJC. This finding was different from a randomized placebo controlled trial that used PTX $400 \mathrm{mg}$ tablets three times daily or placebo for 24 weeks which showed that PTX was more effective than placebo in term of reducing SJC [17]. This difference can be attributed to the short duration of this study in comparison to the above study that continued for 24 weeks.

Assessment of tender points is the cornerstone during evaluation and treatment decision making in RA [18]. This study showed that there was a non-significant difference between the effect of PTX and placebo; similar finding was reported by Maksymowych et al [19] who carried out a prospective 3-month open evaluation of PTX in a group of adult patients with RA refractory to conventional disease remittive therapies and found PTX was effective to reduce TJC in patients with mild active
RA more than placebo but the effect was not statistically significant.

Visual analogue scale (VAS) is a valid measure of pain intensity in RA patients [20]. The current study showed that there was a non-significant difference between the effect of PTX and placebo on VAS. Similar study in which the effect of PTX on painful distal diabetic neuropathy was measured by Cohen et al who used PTX in six-month trial for the treatment of painful distal diabetic neuropathy and found non-significant difference in the patients' pain between the PTX- and placebo-treated groups [21]. But Usha et al [17] reported that PTX reduced VAS significantly while placebo not. The difference may be due to the difference in duration of the present study or the use of etanercept with placebo that were also effective in reducing VAS and may cover the effect of PTX.

Evaluator global assessment (EGA) is considered a reliable indicator for assessing RA and even more reliable than VAS [22]. We found a non-significant difference between the effect of PTX and placebo on EGA. Exactly there were no similar studies in this respect and this was the first study evaluated the effect of PTX on EGA.

Acute phase reactants ESR and CRP provide reliable means for discrimination between drugs that provide symptomatic relief only and those with a more profound effect in RA [23]. In this study there was non-significant difference of the effect of PTX on ESR. In contrast to Maksymowych [19] who found PTX decreased ESR significantly after 3 months. Possible explanation may be 
the effect of etanercept in our patients making effect of PTX much harder to detect.

The present study showed that RA patients had a significantly higher CRP level than healthy control subjects, which was similar to another study that reported a higher CRP level in RA patients than in healthy control subjects and CRP elevation was directly correlated with RA disease activity [24]. The results also showed a significant effect of PTX but not placebo on hsCRP level this result was similar to randomized placebo-controlled study done by Fernande et al [25] who measured proinflammatory and anti-inflammatory activity of PTX in patients with coronary artery disease and concluded that PTX reduced pro-inflammatory CRP level significantly. Another study done by Maitia et al [26] showed after onemonth follow-up PTX decreased significantly C-reactive protein and ESR.

Regarding RA disease activity, PTX had non significant effect on both DAS28 and SDAI. This was the first study that measured the effect of PTX on DAS28 or SDAI and the result can be explained since these measures depend on different factors include TJC, SJC, VAS, ESR, hsCRP so the effect of PTX will be the result of effect on the above factors which showed a high percent of change in PTX group but non significant effect.

The results of this study showed a significant difference between the effect of PTX and placebo on TNF- $\alpha$. Similarly González-Espinoza et al [27] showed that PTX significantly decreased serum concentrations of TNF- $\alpha$, IL-6 and CRP compared to placebo. This may suggest that PTX could be a promising and useful strategy to reduce the systemic inflammation.

Additionally, we found that PTX reduced morning stiffness significantly. Inconsistent with Huizinga et al [28] who found that PTX produced non-significant change in morning stiffness when used in combination with thalidomide. The finding in our study was expected possibly because PTX could improve the markers of inflammation that morning stiffness was correlated with like ESR, swollen joint counts, pain, fatigue, tender joint and patient and physician global assessment of disease activity [29]. Also the above study included only 12 patients and used PTX as adjuvant with thalidomide which differs in mechanism of action and efficacy from etanercept which was used in current study.

The results of this study showed that Hb level in RA patients was significantly less than control subjects, this finding was proved by many other studies showing that anemia was a common extra articular manifestation of RA patients [30,31]. Also neither PTX nor placebo could improve $\mathrm{Hb}$ level which was similar to another animal study done by Mendes et al who found PTX decreased hemoglobin content by approximately 40\% [32].

Moreover, WBC count was significantly higher in RA patients than control subjects; this finding was similar to another clinical trial that showed a high level of WBC count in RA patients[33] whereas other studies found a correlation between inflammation and WBC count [34]. The data also showed that PTX was no more effective than placebo to decrease WBC count, Inconsistent with animal study done by Mohammadzadeh et al [35] who concluded PTX could decrease total white blood cell count in serum of rats.
Furthermore, PTX produced a significant change in hsCRP and TNF which are the most important markers for $\mathrm{CV}$ risk specially the level of circulating CRP which is a prognostic marker of future $\mathrm{CV}$ events for men and women both with and without established CVD [36].The non-significant effect on other inflammatory parameters that increase cardiovascular risk like ESR may be related to use of other drugs with PTX and placebo that may mask the positive effect of PTX on these CV risk factors. Fernandesa, et al[37] reported PTX reduced the proinflammatory and increased anti-inflammatory response in patients with acute coronary syndrome and may have beneficial clinical effects on cardiovascular events.

In conclusion, PTX significantly decreased proinflammatory markers (TNF, hsCRP), duration of morning stiffness, and cardiovascular risk. This may suggest that PTX could be a promising and useful strategy to reduce the systemic inflammation and cardiovascular morbidity and mortality observed in RA patients.

\section{References}

[1] Christopher VT, Joan MB. Rheumatoid arthritis. In: Klippel JH, Stone JH, Crofford LJ, White PH, eds., Primer on rheumatic diseases, 13th ed., New York, USA, Springer science and business media 2008; 114-42.

[2] Nurmohamed MT. Cardiovascular risk in rheumatoid arthritis. Autoimmun Rev 2009;8:663-7.

[3] Avina-Zubieta JA, Choi HK, Sadatsafavi M, Etminan M, et al. Risk of cardiovascular mortality in patients with rheumatoid arthritis: a meta-analysis of observational studies. Arthritis Rheum 2008; 59:1690-7.

[4] Bradley JR. TNF-mediated inflammatory disease. J Pathol 2008; 214(2):149-60.

[5] "P” Monographs; Pentoxifylline (Systemic). In: Group UDE, ed. USP DIR Drug Information for the Health Care Professional, 26th ed. Taunton, MA: Micromedex, 2006.

[6] Bayat M, Chelcheraghi F, Piryaei A, RakhshanMet al. The effect of 30-day pretreatment with Pentoxyphylline on the survival of a random skin flap in the rat: an ultrastructural and biomechanical evaluation. Med SciMonit 6 (2006). 12(6): 201-207

[7] Romy Beatriz, Christmann de Souza, André Regis Macedo „Kátia Akemi Kuruma. Pentoxyphylline in association with vitamin E reduces cutaneous fibrosis in systemic sclerosis ClinRheumatol (2009) 28:1207-1212.

[8] Maksymowych WP, Avina-Zubieta A, Luong MH, Russell AS. An open study of pentoxifylline in the treatment of severe refractory rheumatoid arthritis. J Rheumatol. 1995 Apr; 22(4):6259.

[9] Huizinga TW, Dijkmans BA, van der Velde EA, An open study of pentoxifyllineand thalidomide as adjuvant therapy in the treatment of rheumatoid arthritis. Annals of the Rheumatic Diseases, 1996; 55(11):833-836

[10] Ishii O, Yamada H, Ohya S, Moriuchi E, et al. Remission induction after pentoxifylline treatment in a patient with rheumatoid arthritis. Ryumachi, 1997 Dec; 37(6):810-5.

[11] RaghdanZeki Al-Saad, SaadAbdulrahmanHussain and Intesar Tariq Numan. Dose-response Relationship of the Antiinflammatory Activity of pentoxifylline.

[12] Stojanov S, Kastner DL. Familial auto inflammatory diseases: genetics, pathogenesis and treatment. CurrOpinRheumatol 2005; 17: 586-599.

[13] Robert Landewe. Barriers to Effective Disease Activity Monitoring in Rheumatoid Arthritis - Clinical Practice and Implications for Treat-to-target Guidelines. European Musculoskeletal Review, 2011;6(2):88-93.

[14] Mitra B, Panja M. High sensitive C-reactive protein: a novel biochemical markers and its role in coronary artery disease, $\mathrm{J}$ Assoc physicians India .2005 Jan; 53:25-32

[15] So T, Lee SW, Croft M. Tumor necrosis factor/ tumor necrosis receptor family members that positively regulate immunity. Int $\mathrm{J}$ Hematol.2006; 83(1):1-11. 
[16] Soubrier M, Zerkak D, Gossec L, et al. Which variables best predict change in rheumatoid arthritis therapy in daily clinical practice? J Rheumatol 2006; 33(7):1243-6.

[17] Usha P R, Naidu M U R, Datla R. Clinical Efficacy and Tolerability Evaluation of pentoxifylline in Rheumatoid Arthritis: A Double-Blind, Randomised, Placebo-Controlled Study. Clinical Drug Investigation, 2002; 22(5): 329-339.

[18] Terence Starz. Tender Points in Rheumatoid Arthritis - How Do They Help Us? J Rheumatol 2012; 39(1):1-3.

[19] Maksymowych WP, Avina-Zubieta A, Luong MH, Russell AS. An open study of pentoxifylline in the treatment of severe refractory rheumatoid arthritis. The Journal of Rheumatology .1995; 22(4):625-629.

[20] Richard Charles John Campbell, Michael Batley, et al. The impact of disease activity, pain, disability and treatments on fatigue in established rheumatoid arthritis. April 2012, Volume 31, Issue 4, pp 717-722.

[21] Steven M. Cohen. Pentoxifylline in the Treatment of Distal Diabetic Neuropathy. Angiology September 1991 vol. 42 no. 9 741-746.

[22] Rohekar G, Pope J. Test-retest reliability of patient global assessment and physician global assessment in rheumatoid arthritis. J Rheumatol. 2009; 36(10):2178-82. Epub 2009 Sep 15

[23] Amos RS, Constable TJ, Crockson RA, et al. Rheumatoid arthritis: relation of serum C-reactive protein and erythrocyte sedimentation rates to radiographic changes. Br Med J. 1977; 1(6055): 195-7.

[24] Yildirim K, Karatay S, Melikoglu MA, et al. Associations between Acute Phase Reactant Levels and Disease Activity Score (DAS28) in Patients with Rheumatoid Arthritis. Ann Clin Lab Sci. 2004;34(4):423-6.

[25] Fernandes JL, de Oliveirab R T, R L Mamoni R L, et al. Pentoxifylline reduces pro-inflammatory and increases antiinflammatory activity in patients with coronary artery disease- $\mathrm{A}$ randomized placebo-controlled study. Atherosclerosis. January 2008; 196 (1): 434-42.

[26] Maiti R, Agrawal NK, Dash D, Pandey BL. Effect of pentoxifylline on inflammatory burden, oxidative stress and platelet aggregability in hypertensive type 2 diabetes mellitus patients .VasculPharmacol. 2007 Aug-Sep;47(2-3):118-24.

[27] González-Espinoza L, Rojas-Campos E, Medina-Pérez M, et al. pentoxifylline decreases serum levels of tumor necrosis factor alpha, interleukin 6 and C-reactive protein in hemodialysis patients: results of a randomized double-blind, controlled clinical trial. Nephrol Dial Transplant. 2012 May; 27(5):2023-8.

[28] Huizinga TW, Dijkmans BA, van der VeldeEA ,et al. An open study of pentoxifylline and thalidomide as adjuvant therapy in the treatment of rheumatoid arthritis .Ann Rheum Dis 1996; 55: 833-6.

[29] Fiehn C, Kessler S, Müller K. Morning stiffness of the joints is the sole predictor of short-term response to glucocorticoid treatment in active rheumatoid arthritis (RA). Rheumatol Int. 2012 Dec; 32(12):4069-70.

[30] Manole C, Cojocaru I M, Isabela S, CameliaDoinaVrabie, et al. Extra-articular manifestations in Rheumatoid Arthritis. Maedica (Buchar) 2010; 5(4):286-291.

[31] Cimmino MA, Salvarani C, Macchioni P, Montecucco C, et al. Extra-articular manifestations in 587 Italian patients with rheumatoid arthritis. RheumatolInt 2000; 19(6):213-7.

[32] Mendes JB, Campos PP, Rocha MA, Andrade SP. Cilostazol and pentoxifylline decrease angiogenesis, inflammation, and fibrosis in sponge-induced intraperitoneal adhesion in mice. Life Sci. 2009 Apr 10; 84(15-16):537-43

[33] Pasupathi P, Deepa M, Rani P, Sankar RR. Circulating lipid peroxidation, plasma and erythrocyte antioxidant status in patients with rheumatoid arthritis. Bangladesh Med Res Counc Bull. 2009 Aug; 35(2):57-62.

[34] Ford ES. The metabolic syndrome and C-reactive protein, fibrinogen, and leukocyte count: findings from the Third Nationa Health and Nutrition Examination Survey. Atherosclerosis 2003; 168(2):351-8.

[35] Mohammadzadeh A, Heidari M, SoltanghoraeeH,et al. Evaluation of the effect of pentoxifylline on white blood cell count in serum and peritoneal fluid in female rats with endometriosis. J ObstetGynaecol Res. 2008 Jun; 34(3):307-13.

[36] Lukasz Kozera, Jacqueline Andrews, Ann W Morgan. Differentiating True Soluble Biomarkers of Cardiovascular Risk From Surrogate Measures of Inflammation Rheumatol 2011; 50(11):1944-1954.

[37] Fernandes JL, de Oliveirab $\mathrm{R}$ T, R L Mamoni R L, et al. pentoxifylline reduces pro-inflammatory and increases antiinflammatory activity in patients with coronary artery disease-A randomized placebo-controlled study. Atherosclerosis. January 2008; 196 (1): 434-42. 